\title{
GOCE gradients in various reference frames and their accuracies
}

\author{
J. Müller \\ Institut für Erdmessung (IfE), University of Hannover, Schneiderberg 50, D-30167 Hannover, Germany
}

\begin{abstract}
The objective of GOCE (Gravity Field and Steady-State Ocean Circulation Explorer) is the determination of the Earth's gravity field with high spatial resolution. The main science sensor (the gradiometer) measures differential accelerations, from which the gravitational gradients, i.e. the matrix of the second derivatives of the gravitational potential, are derived. Some of them (the diagonal components of the gravitational tensor) are observed with highest accuracy, $4 \mathrm{mE} / \sqrt{\mathrm{Hz}}$ in a frequency range from $5 \mathrm{mHz}$ to $100 \mathrm{mHz}$, whereas the off-diagonals are obtained less accurately.

The gradients will be observed in the instrument frame, which approximates the along-track oriented, local orbital frame. For the transformation of the gradients in other frames (e.g. in the strictly earth-pointing frame or a local geodetic frame), the transformation parameters (orientation angles) and all components of the gravity tensor have to be known with sufficient accuracy. We show how the elements of the gravitational tensor and their accuracies look like in the various frames as well as their spectral behaviour, if only the GOCE observations are used for the transformation.

Only $V_{z z}^{\prime}$ keeps approximately its original accuracy in all frames discussed, except in the earth-fixed frame ITRF (International Terrestrial Reference Frame). Therefore we recommend to analyse the gradients as 'close' as possible in the observation frame.
\end{abstract}

Key words. Satellite gradiometry, GOCE mission, reference frames, transformation errors

\section{Introduction}

The objective of GOCE is the determination of the Earth's gravity field with high spatial resolution (about $100 \mathrm{~km}$ on the surface of the Earth) and with a high and homogeneous accuracy, i.e. aiming to a geoid error of about $1 \mathrm{~cm}$ up to a spherical harmonic degree $l=200$. GOCE applies Satellite

Correspondence to: J. Müller (mueller@ife.uni-hannover.de
Gravity Gradiometry for the derivation of the medium/shortwavelength parts and Satellite-to-Satellite Tracking with the GPS satellites for the orbit determination and the retrieval of the long-wavelength part of the gravity field (see also ESA, 1999).

The gravitational gradients will be observed in the instrument frame, which approximates the along-track oriented, local orbital frame (beside some misalignments). For the transformation of the gradients in other frames, e.g. in the strictly earth-pointing frame, which differs from the alongtrack oriented by a small rotation about the cross-track axis, or in a local geodetic (also called geographical) frame, the transformation parameters (orientation angles) and the various components of the gravitational tensor have to be known with sufficient accuracy. As the accuracy of both quantities is restricted because of instrumental or conceptual reasons, the resulting gradients in the new frames have only a reduced accuracy. We have investigated how the errors are propagated into the frames under discussion, where we have only worked with the 'raw' GOCE observations, i.e. gradients, orbits and angles (without further pre-processing to posssibly reduce the effect of the less accurate off-diagonals). The results are shown in the spectral domain.

Where we needed simulated gradients and GOCE positions, we took the test data set provided by the IAG SC7 (see the corresponding web page: http://www.geod.uni-bonn.de/ SC7/index.html). This data set is sampled with a frequency of $0.2 \mathrm{~Hz}$, in contrast to the real GOCE mission, where $1 \mathrm{~Hz}$ is planned. Attitude errors were computed with the GOCE gradiometer simulator of H. Oberndorfer (private communication; see also Smit et al., 2000).

\section{GOCE gradiometry}

The GOCE gradiometer is realised by an ensemble of six three-axes accelerometers in the so-called diamond configuration (see Fig. 1). One axis of each accelerometer indicated by the dots is less sensitive than the others, which has instru- 


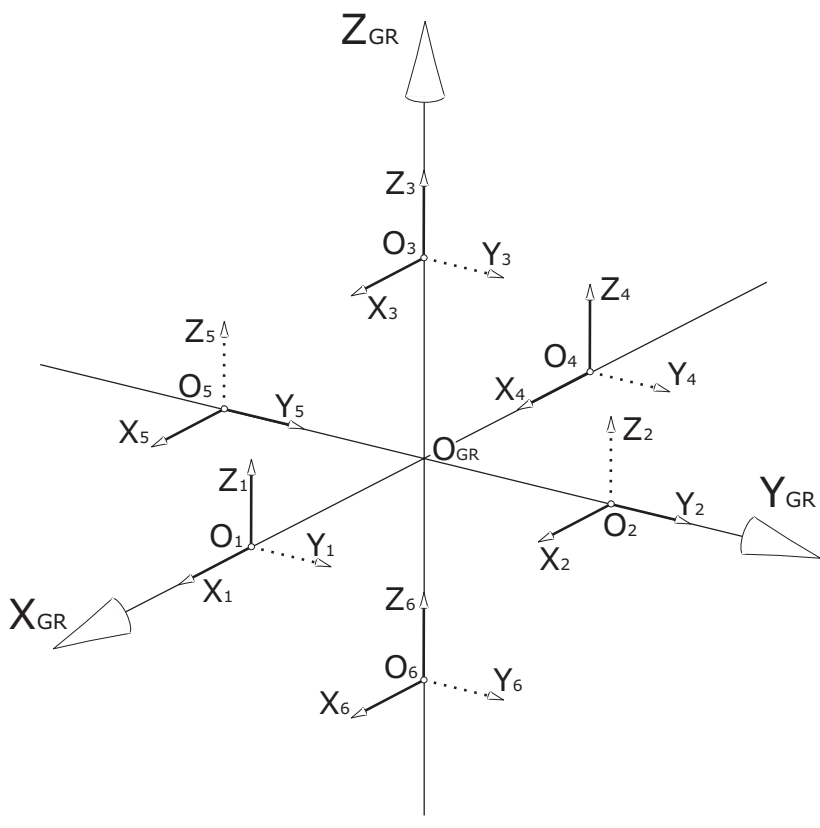

Fig. 1. The GOCE gradiometer with six three-axes accelerometers in the so-called diamond configuration. The dots indicate the less sensitive axes.

mental reasons, and depends also on the pre-flight calibration process.

The real observations are differential accelerations (in principle these are voltages scaled to accelerations) over short baselines. For example, the differential acceleration in $x$-direction is obtained as (see also Müller and Oberndorfer, 1999)

$\Gamma_{x x}=\frac{a_{1}^{x}-a_{4}^{x}}{d^{x}}$,

where $a_{1}^{x}$ and $a_{4}^{x}$ are the accelerations along the $x$-axis measured by the accelerometers 1 and $4 ; d^{x}$ is the distance between the accelerometers. In general, the acceleration at a position $\mathbf{r}$ in a rotating reference frame is defined by

$\frac{D^{2} \mathbf{r}}{D t^{2}}=\left(a^{x}, a^{y}, a^{z}\right)^{t}=\mathbf{F}+(\mathbf{V}-\mathbf{\Omega} \mathbf{\Omega}+\dot{\mathbf{\Omega}}) \mathbf{r}$,

where the Coriolis term has been neglected because the accelerometer proof mass is kept in rest. $\mathbf{F}$ represents the sum of all non-gravitating accelerations, which are mainly caused by air drag and thruster firing; $\mathbf{r}$ gives the position of the proof mass with respect to the satellite's center of mass. The tensor $\mathbf{V}$ is given by the second derivatives of the Earth's gravitational potential:

$\mathbf{V}=\left(\begin{array}{ccc}V_{x x} & V_{x y} & V_{x z} \\ V_{y x} & V_{y y} & V_{y z} \\ V_{z x} & V_{z y} & V_{z z}\end{array}\right)$

This tensor is symmetric and trace-free. $\boldsymbol{\Omega} \boldsymbol{\Omega}$ and $\dot{\boldsymbol{\Omega}}$ describe the effect of the inertial forces onto the observations. They

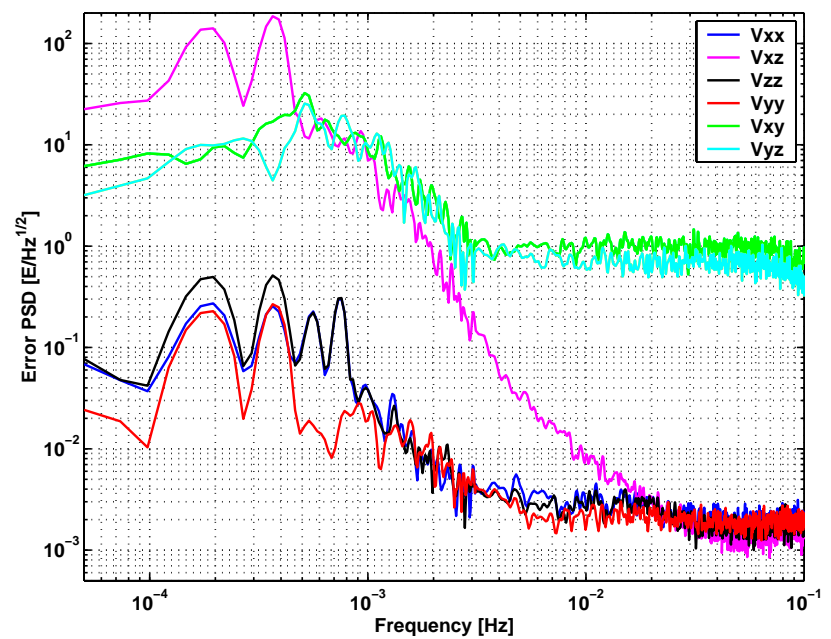

Fig. 2. Error PSD of the essential matrix elements of the gravitational tensor $\mathbf{V}$. Only the in-line elements keep the full accuracy of $4 \mathrm{mE} / \sqrt{\mathrm{Hz}}$ over the whole measurement bandwidth from $5 \mathrm{mHz}$ to $100 \mathrm{mHz}$.

can be computed from the angular velocities $\boldsymbol{\Omega}$ and their time derivatives, the angular accelerations:

$\boldsymbol{\Omega} \boldsymbol{\Omega}=\left(\begin{array}{ccc}-\Omega_{y}^{2}-\Omega_{z}^{2} & \Omega_{x} \Omega_{y} & \Omega_{x} \Omega_{z} \\ & -\Omega_{x}^{2}-\Omega_{z}^{2} & \Omega_{y} \Omega_{z} \\ \text { symm. } & & -\Omega_{x}^{2}-\Omega_{y}^{2}\end{array}\right)$,

$\dot{\mathbf{\Omega}}=\left(\begin{array}{ccc}0 & \dot{\Omega}_{z} & -\dot{\Omega}_{y} \\ -\dot{\Omega}_{z} & 0 & \dot{\Omega}_{x} \\ \dot{\Omega}_{y} & -\dot{\Omega}_{x} & 0\end{array}\right)$.

$\Omega_{x}, \Omega_{y}$ and $\Omega_{z}$ are the angular velocities about the $x$-, $y$ and $z$-axis, respectively; the dots (e.g. like at $\dot{\Omega}_{x}$ ) indicate the time derivative. The matrix $\dot{\boldsymbol{\Omega}}$ is anti-symmetric, whereas $\boldsymbol{\Omega} \boldsymbol{\Omega}$ is symmetric. Calculating all possible differences as indicated in Eq. (1) yields the matrix of the gradiometric observations:

$\Gamma=\mathbf{V}-\mathbf{\Omega} \boldsymbol{\Omega}+\dot{\mathbf{\Omega}}$.

After pre-processing, the matrix of the second derivatives of the gravitational potential $\mathbf{V}$ is obtained. But, as already mentioned, an accuracy of at least $4 \mathrm{mE} / \sqrt{\mathrm{Hz}}$ in a frequency range from $5 \mathrm{mHz}$ to $100 \mathrm{mHz}$ (often called measurement bandwidth MBW) can only be achieved when combining the readouts of two highly sensitive accelerometer axes. Therefore only the diagonal components $\left(V_{x x}, V_{y y}\right.$ and $\left.V_{z z}\right)$ of the gravitational tensor are obtained with sufficient accuracy to be used for gravity field determination. Although the two offdiagonals $V_{x z}$ and $V_{z x}$ are observed with the high-sensitive axes, they do not achieve an accuracy of $4 \mathrm{mE} / \sqrt{\mathrm{Hz}}$ in the whole MBW because the pointing angles are not known with sufficient accuracy, which leads to an insufficiently known projection of the large in-line gradients into the off-diagonal elements. The other off-diagonal second derivatives $\left(V_{x y}\right.$, 

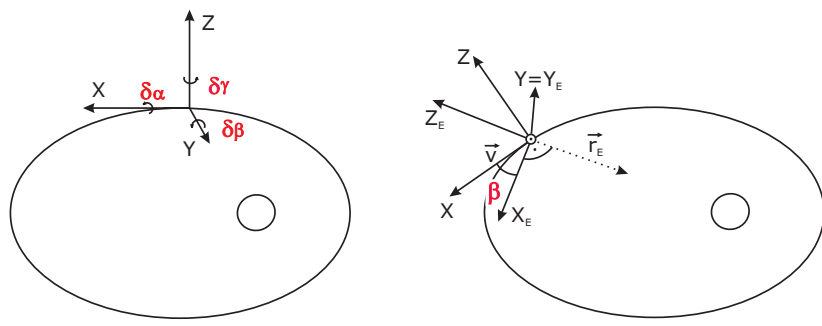

Fig. 3. Left: Orientation of the along-track oriented, local orbital frame where $x$ points along-track, $y$ cross-track and $z$ completes the frame to an orthonormal basis. Right: Strictly earth-pointing, local orbital frame which differs from the along-track oriented one by a small rotation about the cross-track axis in the order of $10^{-3}$ rad.

$V_{y x}, V_{y z}$ and $\left.V_{z y}\right)$ are even worse, mainly caused by combinations of measurements performed with one or more of the less sensitive accelerometer axes. The error Power Spectral Densities (PSDs) of all essential matrix elements of $\mathbf{V}$ are shown in Fig. 2. The contribution of the negative frequencies in the PSDs has already been added to the curves shown. Obviously the high precision in the MBW is only kept by the diagonal elements, but not by the off-diagonals.

The remaining errors in the diagonal matrix elements are mainly caused by the limited precision of the individual sensors (e.g. accelerometers) as well as by the interaction with each other and with the control systems and actuators. Additionally various error sources play a role like instrumental errors which may be scale factor errors, misalignments, the non-orthogonality of the accelerometer axes or the non-linear behaviour of the accelerometer measurement. Therefore the gradiometer measurements have to be calibrated on ground (pre-flight), in orbit and in post-processing to reduce these errors (e.g. Bouman and Koop, 2002).

\section{Reference frames}

The tensor components and their accuracies discussed so far are valid in the instrument frame co-moving with the satellite along the orbit. This reference frame, the along-track oriented, local orbital frame, is defined in the following way: $x$ points in flight direction (along-track), $y$ is orthonormal to the orbital plane (cross-track) and $z$ completes the frame to an orthonormal basis (Fig. 3, left). Because of the orbit eccentricity, $z$ does not point radially to the geocenter. The attitude control system keeps the satellite and thus the gradiometer in this orientation within small deviations $(\delta \alpha, \delta \beta$ and $\delta \gamma)$ of about $10^{-4} \mathrm{rad}$ at zero frequency and $10^{-5} \mathrm{rad} / \sqrt{\mathrm{Hz}}$ in the MBW. These variations are generated by the coupling of the remaining non-gravitational orbit perturbations and the non-ideal thruster behaviour.

In the further processing the gradiometer observations (gravitational gradients) might be used in other frames, e.g. if the gradients shall be combined with terrestrial data (e.g.
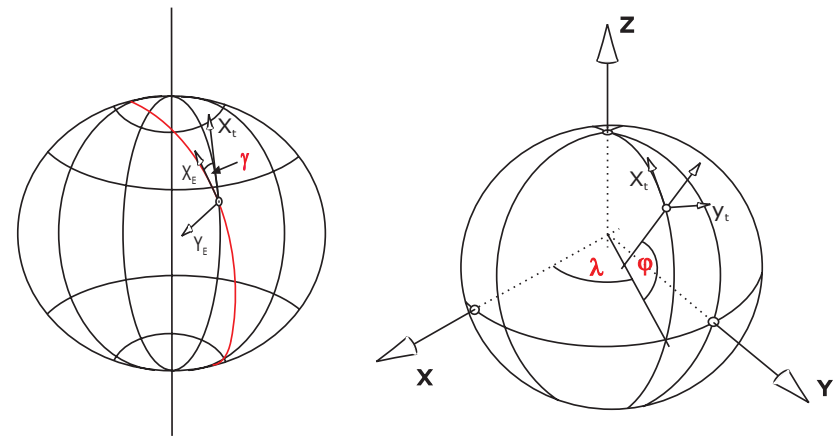

Fig. 4. Left: Local geodetic (or geographical) frame where $x_{t}$ points towards North, $y_{t}$ to East and $z_{t}$ upwards and its relation to the earth-pointing, local orbital frame. Right: Relation between local geodetic frame and the global earth-fixed frame, e.g. ITRF2000.

gravity anomalies), which are normally given in a local geodetic reference frame - often also called geographical frame - where one axis points towards North, one to East and the third upwards (Fig. 4, left). Note, we have used this frame together with the geocentric latitude (instead of the ellipsoidal latitude), not to complicate the transformations too much. Or some user may need gravity field quantities in an intermediate frame (e.g. on a sphere with a certain radius). Also the Earth gravity field itself is defined in another frame, the earth-fixed frame (Fig. 4, right). In this paper, we investigate which accuracies the gradients may have in various frames, if one just uses the raw GOCE observations (i.e. gradients and angles) for the transformation. In detail, we have computed gradients in the strictly earth-pointing frame (Fig. 3, right), which differs from the aforementioned alongtrack oriented, local orbital frame by a small rotation about the cross-track axis in the order of $10^{-3} \mathrm{rad}$, which is caused by the orbit eccentricity. Furthermore we consider the local geodetic frame (North, East, up) and the earth-fixed frame (e.g. the ITRF2000) where one axis points to the North pole, one to Greenwich and the third completes the system.

\section{Transformation of gradients and errors}

Beginning with the gravitational gradients in the along-track oriented, local orbital frame, we have computed the gradients and their errors in other reference frames which have been described in the previous section. The transformation of the matrix of the gravitational gradients between two reference frames can be achieved by

$\mathbf{V}^{\prime}=\boldsymbol{\Theta}^{t} \mathbf{V} \boldsymbol{\Theta}$.

$\mathbf{V}^{\prime}$ is the gravitational tensor in the new frame (after rotation) and $\mathbf{V}$ the originally observed one:

$\mathbf{V} \approx\left(\begin{array}{ccc}1370 & 1 & 10 \\ & 1370 & 2 \\ \text { symm. } & & -2740\end{array}\right) \mathrm{E}$. 


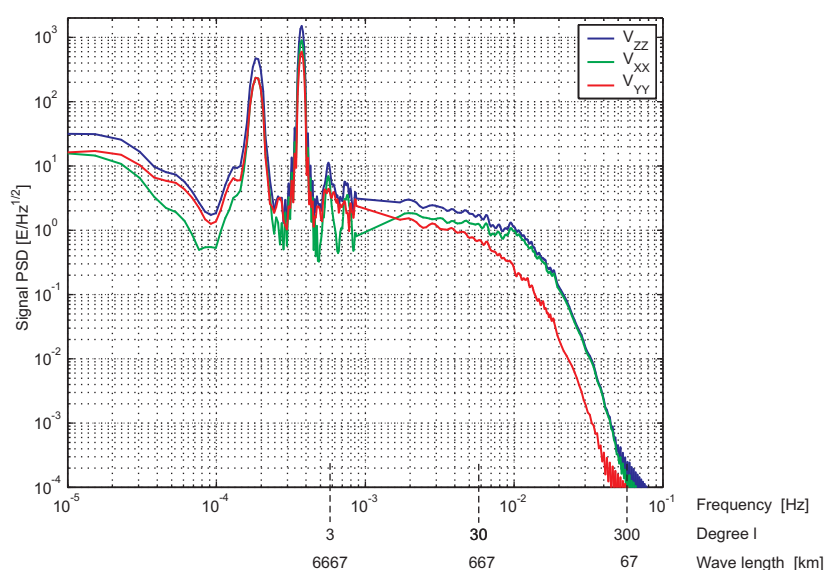

Fig. 5. Signal PSD of the diagonal elements of the gravitational tensor $\mathbf{V}$.

$\mathrm{E}=10^{-9} \frac{1}{\mathrm{~s}^{2}}$ is the Eötvös unit. The numbers in Eq. (8) indicate mean values, i.e. they represent the signal values at zero frequency. The spectral behaviour of the in-line gravity gradients of $\mathbf{V}$ is shown in Fig. 5. The variations of $\mathbf{V}$ are about $1 \mathrm{E} / \sqrt{\mathrm{Hz}}$ in the $\mathrm{MBW}(5 \mathrm{mHz}-100 \mathrm{mHz})$ at maximum, but much larger below MBW. The off-diagonals (not shown here) look similar in the MBW, but not at zero frequency, where they reach the values given in Eq. (8).

$\boldsymbol{\Theta}$ is the rotation matrix (consisting of three rotations about three axes)

$\boldsymbol{\Theta}=R_{3}\left(\vartheta_{z}\right) R_{2}\left(\vartheta_{y}\right) R_{1}\left(\vartheta_{x}\right)$

where $\vartheta_{i}$ are the rotations about the along-track $(x)$, crosstrack $(y)$ and approximately 'radial' $(z)$ direction for the rotation from the along-track oriented satellite frame (Fig. 3, left) to the radially, i.e. strictly earth-pointing frame (Fig. 3, right). Note: Even for small angles of about $1.5 \cdot 10^{-3} \mathrm{rad}$, it would not be allowed to use Eq. (9) in linear approximation because then the accuracy requirements are not met; e.g. $V_{z z^{\prime}}$ would become wrong by about $6 \mathrm{mE}$.

From Eq. (7), it is obvious that the errors of the gradients in the new frames may have two possible origins: the measurement errors of the gradients (one has more and less sensitive accelerometer axes!) and errors of the rotational angles. The latter depend on the errors from the star sensor observations, from the attitude control system (ACS) and from the orbit determination. Time series of the GOCE orbit and the GOCE gradients were provided by IAG SC7. The errors of the gradients are shown in Fig. 2. The rotational errors from the ACS are in the order of about $10^{-4} \mathrm{rad}$ at zero frequency and $10^{-5} \mathrm{rad} / \sqrt{\mathrm{Hz}}$ in the MBW. For the following computations we have assumed, that the errors from the orbit determination are not larger than those from the ACS. Spectral errors in the various frames have been obtained by rigorous error propagation based upon Eq. (7) together with time series of the gradients and their errors as well as those of the transformation angles as mentioned above. For the representation of the

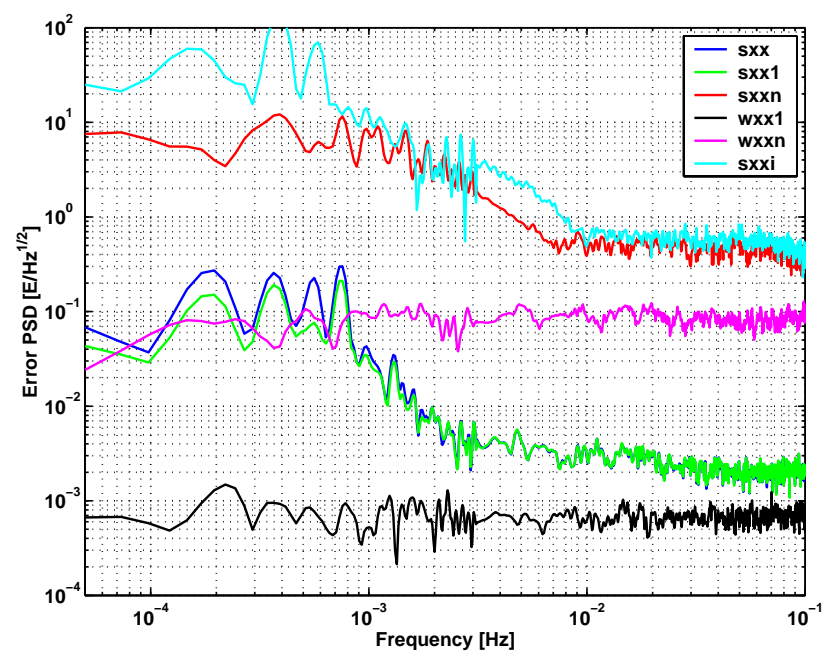

Fig. 6. The error PSDs of $V_{x x}^{\prime}$ in the various frames discussed. ' $s$ ' indicates the errors from the gradients and ' $w$ ' from the angle; ' 1 ' represents the strictly earth-pointing, local orbital frame, ' $n$ ' the geographical (i.e. local geodetic) frame and ' $i$ ' the earth-fixed frame ITRF.

results, Power Spectral Densities (PSD) have been computed from the time series using standard procedures (for more details see e.g. the corresponding contributions in Smit et al., 2000).

In practice, one could think about a suitable pre-processing of the data, e.g. by removing a known reference gravity field. Then badly observed components could be replaced and large gradient values, which couple with angular errors, could be reduced in advance to keep the numbers and also the resulting errors smaller. Such possible pre-processing strategies are not discussed here. We did just work with the full raw observations.

\section{Computations and results}

In a first step, we have just investigated the behaviour of the $V_{x x}$ component. We have transformed it to the strictly earthpointing, local orbital frame frame (see Fig. 3), then to a geographical oriented (i.e. local geodetic) frame and finally to the earth-fixed frame (i.e. the ITRF). Whereas the first rotation (about the cross-track axis) is only small (about $10^{-3}$ $\mathrm{rad}$ ), the rotation to the geographical frame requires rotations about the new $z$-axis, which is the radial one, with angles between $7^{\circ}$ and $173^{\circ}$. This interval is caused by the choice of a sun-synchronous orbit for GOCE. For a polar orbit the angles would vary between $0^{\circ}$ and $180^{\circ}$. In Fig. 6 , the resulting error PSD curves are shown, where we have indicated the errors from the original gradient errors and the angular errors separately. There ' $s$ ' indicates the errors from the gradients and ' $w$ ' from the angles. Moreover ' 1 ' represents the strictly earth-pointing frame, ' $n$ ' the geographical frame and ' $i$ ' the ITRF. These conventions are kept for all following figures. 


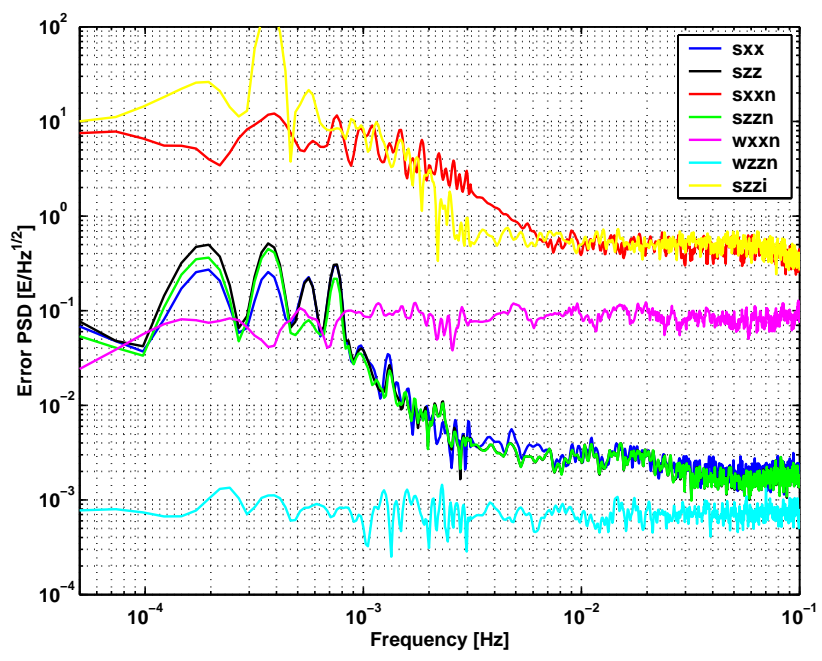

Fig. 7. A comparison of the error PSDs of $V_{x x}^{\prime}$ and $V_{z z}^{\prime}$ in the various frames.

Through the rotations, the 'bad' off-diagonal elements (also shown in Fig. 2) are projected into the new in-line elements. One can also see that in each new frame the main error part comes from the errors of the gradients in the previous step. That means, the errors of the less accurate accelerometer axes have propagated into big errors in the new frames. For example, the green curve 'sxx1' represents the error of $V_{x x}^{\prime}$ in the strictly earth-pointing frame, only caused by the original measurement error of the gradients. It is in the order of the original measurement error, whereas the error contribution from the ACS is less (the black curve 'wxx1'). In the geographical frame the error 'sxxn' (the red curve) of the new $V_{x x}^{\prime}$, which is oriented in the northern direction, becomes almost as high as the signal itself (compare to Fig. 5). The situation becomes even worse, if one transforms to the ITRF, which is indicated by 'sxxi' in Fig. 6. The $V_{y y}^{\prime}$ component behaves similar to $V_{x x}^{\prime}$ and is not shown here explicitly.

We have performed the same computations for all tensor components. Figure 7 shows a comparison between the $V_{x x}^{\prime}$ and $V_{z z}^{\prime}$ components in various frames. The curves for $V_{x x}^{\prime}$ are the same as shown in Fig. 6. $V_{z z}^{\prime}$ behaves differently from $V_{x x}^{\prime}$. It is not affected by the rotation about the radial axis (only small error contributions arise from the rotation from the along-track oriented to the strictly earth-pointing, local orbital frame). Therefore $V_{z z}^{\prime}$ keeps approximately its original measurement accuracy also in the geographical frame; see the the green curve 'szzn'. For $V_{z z}^{\prime}$ again, the error contribution from the less accurate accelerometer axes (the 'bad' gradients) are the main error source, and not possible errors from the computation of the rotation angles. $V_{z z}^{\prime}$ only becomes worse when the transformation to the ITRF is performed because then rotations about big angles (up to $360^{\circ}$ ) are necessary. Finally, Fig. 8 indicates the errors which all tensor components of $\mathbf{V}^{\prime}$ may have in the geographical frame. Only $V_{z z}^{\prime}$ keeps its original accuracy; other tensor elements

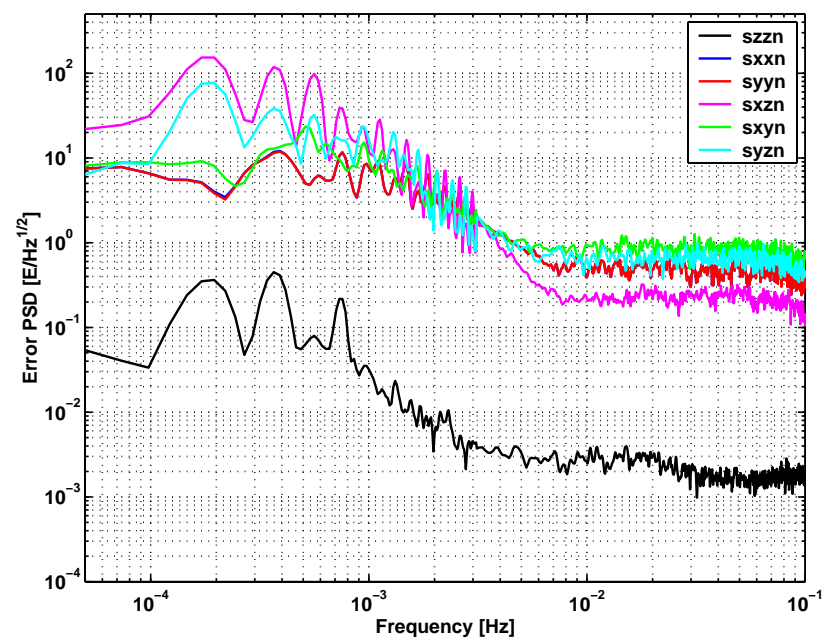

Fig. 8. The error PSDs of all tensor components of $\mathbf{V}^{\prime}$ in the geographical (i.e. the local geodetic) reference frame.

become worse, the errors are in the order of the signal itself.

Our investigations have shown that one has to be very careful if one just takes the 'raw' GOCE measurements (gradients and angles) and transforms them to other reference frames. In most cases the new gradients have a much bigger error, which is mainly caused by the projection of the less accurate off-diagonals into the new tensor elements. $V_{x x}^{\prime}$ and $V_{y y}^{\prime}$ keep their original accuracies only in the local orbital frames, but not in the local geodetic one. In the latter only $V_{z z}^{\prime}$ keeps its high accuracy, but not in the ITRF. Therefore we recommend to analyse the gradients for gravity field determination as 'close' as possible in the local orbital frames.

\section{Conclusion and outlook}

We have investigated how the GOCE gravitational gradients and their errors behave if transformed into various reference frames. We have considered two main error sources: errors from the gradiometer measurements (i.e. which show up in the original gravity gradients) and those from the determination of the rotational angles, which are affected by the ACS and the orbit errors. We have taken into account four different reference frames: two local orbital ones (the along-track oriented and the earth-pointing frame - where the $z$-axis is strictly radial), the local geodetic or geographical frame (North, East, up) and the earth-fixed frame ITRF. Our investigations have shown that the accuracies of the gradients are strongly affected by the errors discussed, where the errors from the propagation of the less acccurate GOCE gradients dominate. We recommend to analyse the gradients as 'close' as possible in the observation frame, i.e. in one of the local orbital frames. Only $V_{z z}^{\prime}$ keeps its original accuracy also in the geographical frame, but not in the ITRF.

If better off-diagonals were needed (e.g. for frame transformations or other applications) one should compute them 
iteratively from a good gravity field, which has been derived from the good diagonals alone. A further possiblity to keep the effect of the less accurate off-diagonal gradients smaller could be achieved by using a known gravity field in a preprocessing step (this aspect was not further discussed in this paper). For future gradiometric missions, it would be optimal to realise a full tensor gradiometer (with high and equivalent accuracies in all components) to be more independent of frame transformations.

Acknowledgements. We are grateful to the members of the European GOCE community, and we like to thank Helmut Oberndorfer (Institute of Astronomical and Physical Geodesy, Technical University Munich) for providing the angular errors computed with his GOCE gradiometer simulator.

\section{References}

Bouman J. and Koop R.: Geodetic Methods for Cal/Val of GRACE and GOCE, Proceedings of the ISSI Workshop about Earth Gravity Field from Space - from Sensors to Earth Sciences, held 11-15 March 2002 in Berne, to be published, 2002.

ESA: Gravity Field and Steady-State Ocean Circulation Explorer, Reports for Assessment: The Four Candidate Earth Explorer Core Missions, ESA SP-1233(1), ESA Publications Division, ESTEC, Noordwijk, 1999.

Müller J. and Oberndorfer H.: Simulation of the Gradiometer Mission GOCE, Artificial Satellites, 34, 1, 41-55, 1999.

Smit M., Koop R., Visser P., van den IJssel J., Sneeuw N., Müller J., and Oberndorfer H.: GOCE End-to-End Performance Analysis, Final Report, ESTEC Contract No. 12735/98/NL/GD, 2000. 